\title{
Dynamic and Stagnating Plasma Flow Leading to Magnetic-Flux-Tube Collimation
}

\author{
S. You, G. S. Yun, and P. M. Bellan \\ California Institute of Technology, Pasadena, California 91125, USA
}

(Received 6 February 2005; published 22 July 2005)

\begin{abstract}
Highly collimated, plasma-filled magnetic-flux tubes are frequently observed on galactic, stellar, and laboratory scales. We propose that a single, universal magnetohydrodynamic pumping process explains why such collimated, plasma-filled magnetic-flux tubes are ubiquitous. Experimental evidence from carefully diagnosed laboratory simulations of astrophysical jets confirms this assertion and is reported here. The magnetohydrodynamic process pumps plasma into a magnetic-flux tube and the stagnation of the resulting flow causes this flux tube to become collimated.
\end{abstract}

The extreme collimation of astrophysical jets [1-3] and the solar corona heating mechanism [4] are two seemingly unrelated astrophysical mysteries, yet both involve collimation of magnetic-flux tubes. Astrophysical observations $[2,3]$ and simulations [1,5] indicate that bipolar plasma outflows (jets) are natural $[1,6]$ features of young stellar objects, black holes, active galactic nuclei, and even aspherical planetary nebula [7]. Although it has long been presumed $[8,9]$ that astrophysical jets are magnetohydrodynamically driven, the standard models do not agree on a single collimation process. A similar issue exists in solar physics: solar spicules [10], prominences [11,12], and coronal loops [13] are considered to be plasma-filled filamentary magnetic-flux tubes; coronal heating models $[14,15]$ then invoke magnetic reconnection and plasma flow within such filamentary loops. However, the models explain neither the origin of the observed flows nor the extreme collimation (filamentary nature) of the observed structures.

We propose that the collimation of any, initially flared, current-carrying magnetic-flux tube is due to the following process [16]: a magnetohydrodynamic (MHD) force resulting from the flared current profile drives axial plasma flows along the flux tube; the flows convect frozen-in magnetic flux from strong magnetic field regions to weak magnetic field regions; flow stagnation then piles up this embedded magnetic flux, increasing the local magnetic field and collimating the flux tube via the pinch effect. Thus, the flux tube fills with ingested plasma and simultaneously becomes collimated. This Letter presents direct experimental evidence for this process. We use ultrahigh-speed imaging and Doppler measurements of the fast plasma flows, combined with direct density measurements before and after the filling of the flux tube.

Our experimental setup [17] simulates magnetically driven astrophysical jets at the laboratory scale by imposing boundary conditions analogous to astrophysical jet boundary conditions (Fig. 1): a disk (cathode) representing a central object such as a star, is coaxial and coplanar with an annulus (anode) representing an accretion disk. A vacuum poloidal magnetic field produced by an external coil links these two electrodes, mimicking a poloidal magnetic field threading the accretion disk. A radial electric field applied across the gap between the disk and annulus drives poloidal current.

The nominal parameters of the laboratory experiment include a plasma discharge duration $\sim 20 \mu \mathrm{s}$, poloidal magnetic field $B \sim 0.01-0.3 \mathrm{~T}$, gun voltage $V_{\text {gun }} \sim 1-7 \mathrm{kV}$, poloidal current $I \sim 50-200 \mathrm{kA}$, and density $\sim 10^{20-22} \mathrm{~m}^{-3}$. The Alfvén speed is $v_{\mathrm{A}} \sim 10^{5} \mathrm{~m} / \mathrm{s}$, and the ion gyroradius is $\sim 0.5 \mathrm{~mm}$, much smaller than the typical scale lengths $(L \sim 1-50 \mathrm{~cm})$. The plasma $\beta$ ranges from $\ll 1$ to $\sim 0.5$, assuming a plasma temperature $\sim 10 \mathrm{eV}$, and the Lundquist number $S=\tau_{r} / \tau_{\mathrm{A}} \sim 10^{3}-10^{5}$, depending on the characteristic length used to define the resistive time $\tau_{r}$ (estimated from classical Spitzer resistivity) and the Alfvén time $\tau_{\mathrm{A}}$. These dimensionless numbers are comparable to numerical MHD simulations.

Just before breakdown, neutral gas is puffed into the vessel from nozzles located on the disk and the annulus.

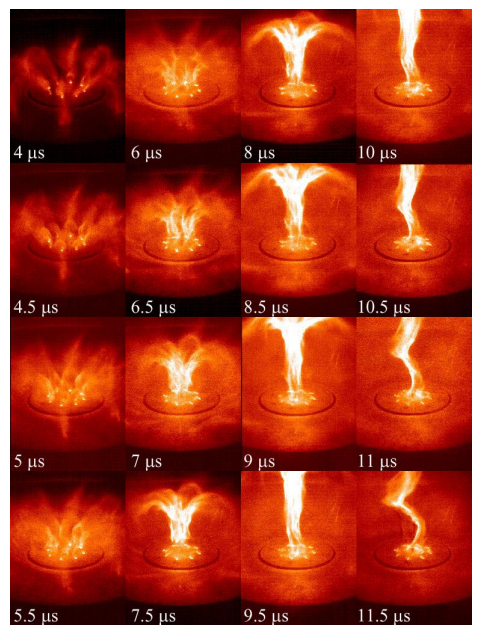

FIG. 1 (color online). Typical plasma discharge sequence (\#6577, $2 \times 10^{6} \mathrm{fps}, 40 \mathrm{~ns} /$ frame, log. color table). Eight collimated "spider legs" merge on axis to form a central column jet that collimates and propagates into the vacuum vessel. 


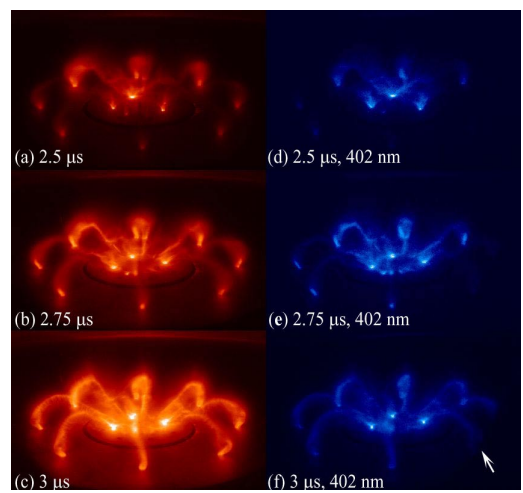

FIG. 2 (color online). Images from identical discharges. (a),(d) 4345; (b),(e) 4346; (c),(f) 4343. Nitrogen is puffed from the cathode nozzles and neon from the outer nozzles. Images (d)-(f) taken simultaneously with (a)-(c), respectively, using a second camera and $402 \mathrm{~nm}$ filter to image nitrogen-rich plasma. Arrow indicates specific spider leg analyzed in detail in Fig. 7.

After breakdown, plasma arches form, analogous to solar prominence loops [18]. These arches are distributed toroidally, reminiscent of spider legs, with each "leg" linking a gas nozzle on the disk to a corresponding nozzle on the annulus (Fig. 1, $4.0 \mu \mathrm{s}$, and Fig. 2). These magnetically dominated plasma arches are initially flared (i.e., their minor radius increases with distance along their axis). Because the coil-produced poloidal vacuum magnetic field is weaker at the annulus than at the disk, the outer foot point diameter is initially 4-5 times that of the inner foot point. The eight legs quickly become bright and highly collimated: in $0.5 \mu \mathrm{s}$, as the legs fill up with plasma, the outer/inner foot point diameter ratio has been measured to reduce by a factor of 2 (Fig. 3), and can approach unity to within $<20 \%$ (Fig. 4). The legs later merge (Fig. 1, 5.5-7.5 $\mathrm{s}$ ) to form a single central column (Fig. 1, $8.0 \mu \mathrm{s})$, which constitutes the axially expanding "astrophysical jet" (Fig. 1, 8-10 $\mu \mathrm{s}$ ). The central column can eventually kink (Fig. 1, 10-11.5 $\mu \mathrm{s}$ ) and lead to spheromak formation [19]. The initial flaring of the spider legs is just the flaring of the coil-imposed vacuum magnetic field, a known analytic function [20].

Measurements shown in Fig. 5 indicate that the neutral gas density existing at breakdown is totally inadequate to account for the plasma density measured in the spider legs or the central column. The measurements were taken with a custom-built fast ion gauge (FIG), with a $2 \mu$ s response time, absolutely calibrated with a standard commercial ion gauge. In situ measurements of the gas cloud in front of the electrodes were fitted numerically to obtain a complete 3D approximation of the gas output from the nozzles. The numerical fit assumed a linear superposition of gas cones from each nozzle, each with a pressure distribution having an axial exponential drop and a radial Gaussian profile. In particular, these measurements indicate that prior to breakdown, the neutral gas density at the location of a single spider leg is $\sim 10^{17} \mathrm{~m}^{-3}$ (Fig. 5), which is 3-4 orders of
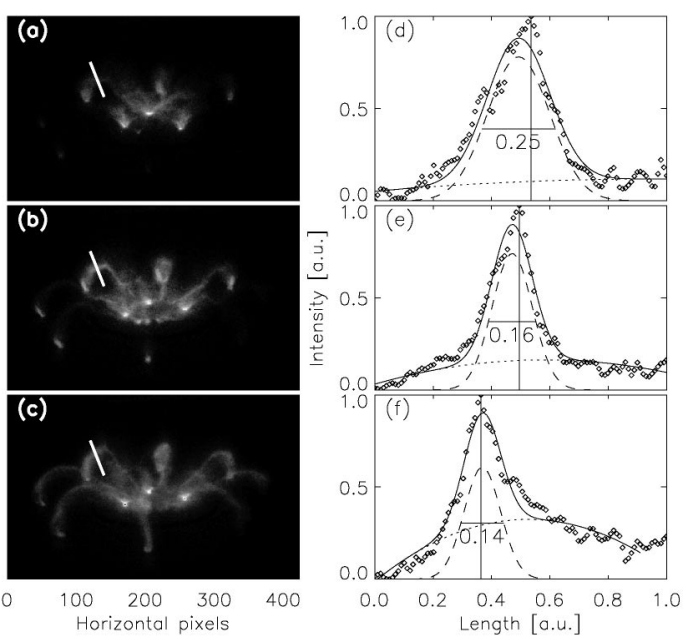

FIG. 3. Spider-leg collimation measurements. (a)-(c) Images from Figs. 2(d)-2(f). Identical white lines indicate the measurements of the spider-leg intensity. (d)-(f) Dotted line shows the quadratic term of the least-squares fit (solid line), dashed line shows the Gaussian term. FWHM (number) is assumed to be representative of the spider-leg width.

magnitude less than the plasma density measured just a few $\mu$ s later [Fig. 6(a)]. These time-resolved FIG measurements also show that, consistent with the molecular thermal velocity of $\sim 1 \mathrm{~km} / \mathrm{s}$, the characteristic time scale for neutral density evolution is $\sim 10 \mathrm{~ms}$, so the neutral gas distribution is quasistationary during the $<50 \mu$ s plasma discharge duration. For a plasma density $10^{3}-10^{4}$ times greater than the locally available supply of neutrals, simple pinching of the tube would require an $\sim(30-100)$-fold decrease in radius. Only a 2 -fold decrease is observed, so all spider-leg particles must be ingested from the gas valves into the leg by MHD forces on $\mu$ s time scales.

To visualize this fast axial flow, nitrogen was injected from the cathode foot points of the spider legs and neon from the anode foot points (Fig. 2). A narrow band inter-

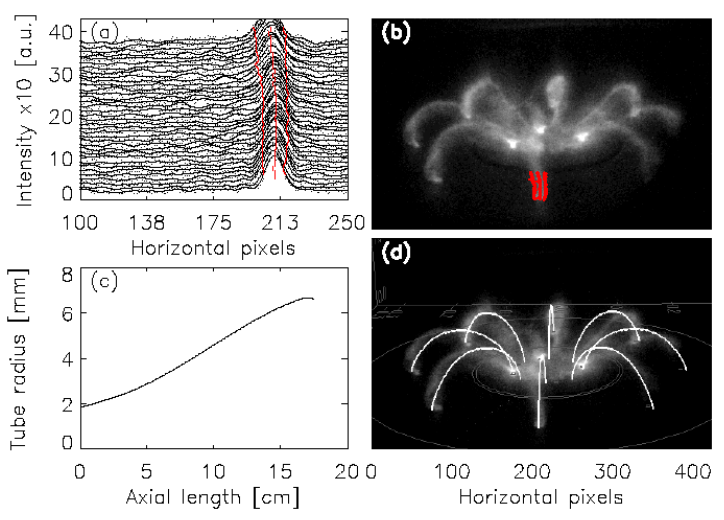

FIG. 4 (color online). (a),(b) Measurement of flux tube flaring, on image 2(f). FWHM (red solid vertical marks) constant to $\lesssim$ $20 \%$. (c) Analytical flaring of flux tube, computed from vacuum poloidal magnetic field [20]. (d) Comparison of the analytical flux tube axis positions (white lines) with image 2(f). 


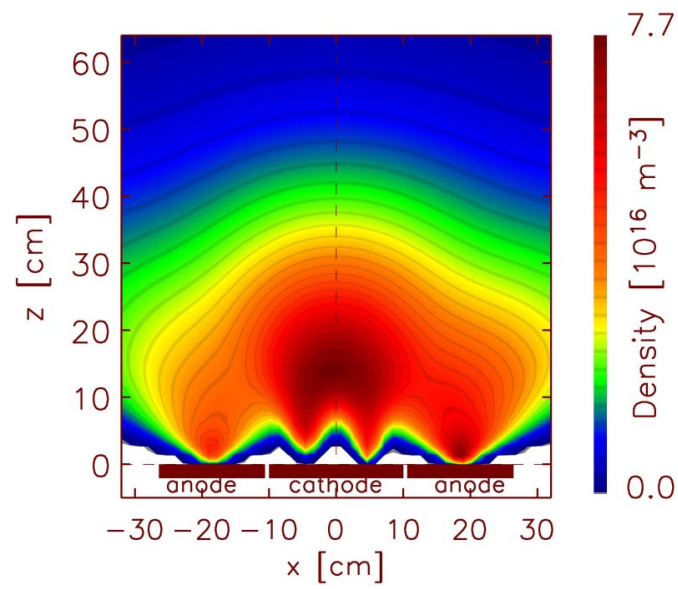

FIG. 5 (color online). Planar section of neutral density distribution just before breakdown, as measured by the fast ion gauge. Dashed line indicates the central axis of the experiment.

ference filter (central wavelength $402 \mathrm{~nm}$, Gaussian passband $10 \mathrm{~nm}$ ) isolated the nitrogen plasma for one camera [Figs. 2(d)-2(f)], while simultaneous, unfiltered photographs were obtained with a second camera [Figs. 2(a)2(c)]. A reconstruction of the experimental geometry overlaid on the images allows comparison of the dimensions of visible legs with best-fit vacuum magnetic-flux tube [Fig. 4(d)]. The photographs indicate a brightness propagating along the axis of the spider-leg flux tube, from the cathode end to the anode end, on a $0.5 \mu$ s time scale (Figs. 2 and 7). This bright front propagation occurs $>1.5 \mu \mathrm{s}$ after the breakdown process has ended, demonstrating that it is different from streamer fronts [21] associated with breakdown mechanisms. The time scale rules out ion acoustic velocities $(8 \mathrm{~km} / \mathrm{s})$ from consideration, and the flow direction rules out electrostatic acceleration. The propagation, in fact, resembles a magnetized vacuum arc discharge [22] and involves nitrogen plasma flow, with $\sim 10^{2} \mathrm{~km} / \mathrm{s}$ velocities (Fig. 7) and a $\sim 10^{11} \mathrm{~m} / \mathrm{s}^{2}$ mean acceleration. Flow velocities can be calculated assuming that the density profile along the axis of the flux tube is proportional to the square root of the light intensity, and integrating the continuity equation
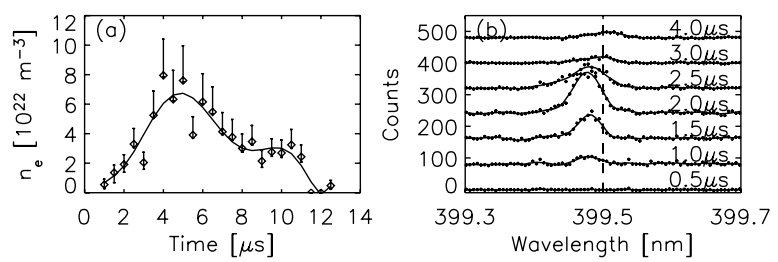

FIG. 6. Spectroscopic measurements: (a) center column "astrophysical jet" $n_{e}(t)$ inferred from Stark broadening. Solid line is a polynomial fit. Errors from 1-10 eV Doppler broadening and differing Lorentzian fit parameters [24]. (b) Doppler shift of single spider leg (N II, $399.5 \mathrm{~nm}$ rest frame, dashed line). Solid lines are polynomial fits. Times correspond to end of $1 \mu \mathrm{s}$ exposure, relative to plasma breakdown.

$$
u(z)=\frac{1}{n(z)} \frac{\partial}{\partial t} \int_{z}^{L} \frac{\partial n\left(z^{\prime}\right)}{\partial t} d z^{\prime},
$$

where the stagnation velocity $u(L)=0$ and $L \sim 18 \mathrm{~cm}$ is the spider leg's axial length (assumed to remain constant during the measurements). The mean acceleration is estimated from the typical velocity and time scale $(u / t \sim$ $10^{5} / 10^{-6} \sim 10^{11} \mathrm{~m} / \mathrm{s}^{2}$ ) or from the typical length scale of the flow $\left[d u_{z} / d t \sim u^{2} /(2 z) \sim 10^{10} /\left(2 \times 5 \times 10^{-2}\right) \sim\right.$ $\left.10^{11} \mathrm{~m} / \mathrm{s}^{2}\right]$.

According to the model [16], the axial component of the MHD pumping force is maximized on the flux tube axis and accelerates plasma along this axis according to the equation of motion

$$
\rho\left(\frac{\partial u_{z}}{\partial t}+u_{z} \frac{\partial u_{z}}{\partial z}\right)=\frac{\mu_{0} I^{2}}{2 \pi^{2} a^{3}} \frac{\partial a}{\partial z},
$$

where $a$ is the local flux tube radius ( $\partial a / \partial z$ is the flaring), $I$ is the total current in the flux tube, $u_{z}$ is the axial plasma velocity, and $\rho$ is the plasma density.

Using spider-leg flux tube dimensions measured from the photographs [Fig. 4(c), $a \sim 4 \mathrm{~mm}, \partial a / \partial z \sim 0.02]$ and assuming the current inside a single leg is one-eighth the total measured $120 \mathrm{kA}$ gun current, Eq. (2) predicts a spider-leg plasma density of $\sim 2 \times 10^{21} \mathrm{~m}^{-3}$. This implies that within $0.5 \mu \mathrm{s}$, MHD forces inject about $2 \times 10^{16}$ nitrogen particles from the gas nozzles into $\mathrm{a} \sim 10 \mathrm{~cm}^{3}$ spider leg. Summing over all eight flux tubes gives a total influx pumping rate of $\sim 3 \times 10^{17}$ particles $/ \mu$ s. Since it takes $\sim 10 \mu$ s for the legs to merge and form the $2.5 \mathrm{~cm}$ diameter, $50 \mathrm{~cm}$ long central column, assuming the pumping rate is constant, the central column should then have a $\sim 1 \times 10^{22} \mathrm{~m}^{-3}$ volume-averaged plasma density. The central column jet, located where $10 \mu \mathrm{s}$ previously there was essentially vacuum, has ingested about $10 \%$ of the total number of particles available from the gas feed lines. At this rate, it would take a few hundred $\mu$ s to empty all the gas from the feed lines. This time is much shorter than the time scale of fusion experiments, where similar filamentary flux tubes have been observed to emerge [23] explosively from the hot plasma core and connect to the cold walls.
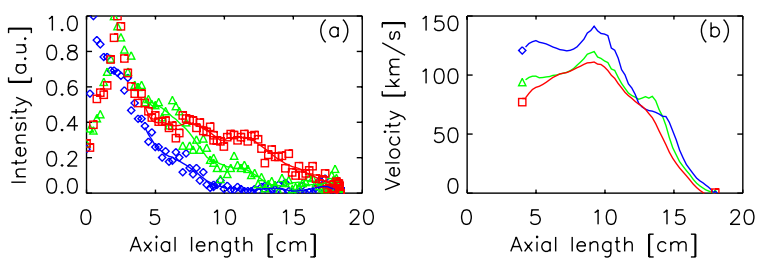

FIG. 7 (color online). Plasma flow measurements: (a) Intensity along axis [of specific spider leg shown in Fig. 2(f)] at $2.5 \mu \mathrm{s}$ [blue diamond, Fig. 2(d)], $2.75 \mu$ s [green triangle, Fig. 2(e)], $3 \mu \mathrm{s}$ [red squares, Fig. 2(f)]. Solid lines are polynomial fits to the data between $4 \leq z \leq 18 \mathrm{~cm}$. (b) Plasma velocities at $2.625 \mu \mathrm{s}$ (blue diamond line), $2.75 \mu \mathrm{s}$ (green triangle line), and $2.875 \mu$ s (red square line) obtained from Eq. (1). 

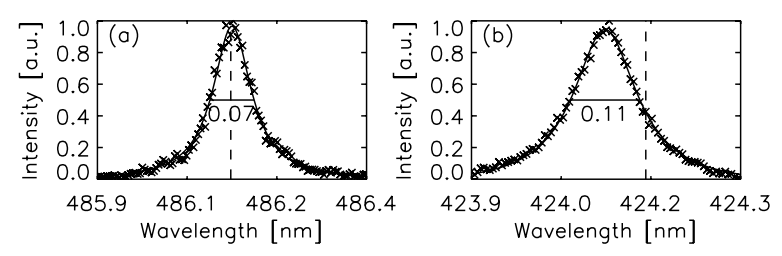

FIG. 8. Measured line spectra with Lorentzian fits: (a) single spider leg, H $486.133 \mathrm{~nm}$ (dashed line). Exposure 0-1 $\mu$ s with respect to breakdown. Line of sight is perpendicular to the spider leg, parallel to the cathode plane. (b) Fully formed central column jet ( $\mathrm{N}$ II, dashed line is $424.178 \mathrm{~nm}$ rest-frame wavelength).

The prediction for very high plasma densities in the spider legs and the central column has been substantiated using independent spectroscopic line measurements [Figs. 6(a) and 8]. These measurements can exhibit large Stark broadening indicative of high electron densities. For hydrogen plasmas, we measure $\sim 2 \times 10^{20} \mathrm{~m}^{-3}$ in the spider leg [Fig. 8(a)] and $\sim 2 \times 10^{22} \mathrm{~m}^{-3}$ in the fully formed central column jet. In nitrogen discharges, central column jet densities $8 \times 10^{22} \mathrm{~m}^{-3}$ are measured [Fig. 6(a) at $4 \mu \mathrm{s}$, Fig. 8(b)]; however, spider-leg densities cannot be resolved. Spectral lines were sampled by optical fibers viewing selected portions of the discharge and recorded with an optical multichannel analyzer. The FWHM $g[\mathrm{~nm}]$ is related to $n_{e}\left[\mathrm{~m}^{-3}\right]$ by $g=1.6 \times 10^{-24} n_{e}$ for N II [24] and $g=2 \times 10^{-15} n_{e}^{2 / 3}$ for $\mathrm{H}$ [25]. The measurements are corrected for the instrument function and a $1-10 \mathrm{eV}$ thermal broadening, estimated from ion line ratios.

The pumping model also predicts that the largest plasma flow velocity in the tube is of the order of the Alfvén velocity associated with the magnetic field generated by the axial current. Here, the $15 \mathrm{kA}$ current, $4 \times 10^{20-21} \mathrm{~m}^{-3}$ density, and $4 \mathrm{~mm}$ typical radius of the spider leg gives $v_{\mathrm{A}} \sim 70-220 \mathrm{~km} / \mathrm{s}$, encompassing the measured $\sim 100 \mathrm{~km} / \mathrm{s}$ shown in Fig. 7. On the axis of a single spider leg [Fig. 6(b)], we observe blueshifts corresponding to $\sim 20-40 \mathrm{~km} / \mathrm{s}$ line-of-sight velocities. Using CCD images, we estimate the optical probe to be $8 \mathrm{~cm}$ along and $1 \mathrm{~cm}$ away from the spider-leg axis, with a line-of-sight angle of $72^{\circ} \pm 3^{\circ}$ to the plasma flow direction. In the central column jet, the flow velocity is measured at $\sim 40-70 \mathrm{~km} / \mathrm{s}$ [Fig. 8(b)], with a line-of-sight coincident to the jet axis. These velocities are consistent with the Alfvén velocity expected from the measured total $150 \mathrm{kA}$ current and $7 \times$ $10^{22} \mathrm{~m}^{-3}$ peak density in the central column. Both the collimation of the spider legs and the central column jet arise from the same process.

The results thus provide strong evidence for flow-driven collimation of flared magnetic-flux tubes. This process requires net electrical current, a supply of particles, and stagnation of the driven flow. In an astrophysical context, accretion disks supply plasma and flows stagnate at the lobes; a net axial current is understood to be necessary for self-confinement [26], and recent observations of helical magnetic field structure in jets are indicative of net axial current [27]. The electrical circuit is then closed by radial currents in the accretion disk, returning via the cocoon. Active solar regions also exhibit unneutralized electrical currents [28]. We emphasize that strong flows and collimation can be driven by even modest amounts of current, before reaching the kink instability (high current) threshold.

This work was supported by the U.S. DoE and NSF.

[1] D. L. Meier, S. Koide, and Y. Uchida, Science 291, 84 (2001).

[2] D.S. De Young, Science 252, 389 (1991).

[3] C. J. Burrows et al., Astrophys. J. 473, 437 (1996).

[4] R. J. Walsh and J. Ireland, Astron. Astrophys. Rev. 12, 1 (2003).

[5] M. Nakamura, Y. Uchida, and S. Hirose, New Astron. Rev. 6, 61 (2001).

[6] D. Lynden-Bell, Mon. Not. R. Astron. Soc. 341, 1360 (2003).

[7] R. Sahai et al., Nature (London) 426, 261 (2003).

[8] R. V. E. Lovelace, Nature (London) 262, 649 (1976).

[9] R. D. Blandford and D. G. Payne, Mon. Not. R. Astron. Soc. 199, 883 (1982).

[10] B. De Pontieu, R. Erdélyi, and S.P. James, Nature (London) 430, 536 (2004).

[11] S. K. Solanki et al., Nature (London) 425, 692 (2003).

[12] E. R. Priest, A. A. van Ballegooijen, and D. H. MacKay, Astrophys. J. 460, 530 (1996).

[13] S. Patsourakos, J. A. Klimchuk, and P. J. McNeice, Astrophys. J. 603, 322 (2004).

[14] E. R. Priest et al., Nature (London) 393, 545 (1998).

[15] M. J. Aschwanden, Astrophys. J. 560, 1035 (2001).

[16] P. M. Bellan, Phys. Plasmas 10, 1999 (2003).

[17] S. C. Hsu and P. M. Bellan, Mon. Not. R. Astron. Soc. 334, 257 (2002).

[18] P. M. Bellan and J.F. Hansen, Phys. Plasmas 5, 1991 (1998).

[19] S. C. Hsu and P. M. Bellan, Phys. Rev. Lett. 90, 215002 (2003).

[20] J.D. Jackson, Classical Electrodynamics (J. Wiley \& Sons, New York, 1975), 2nd ed., p. 178.

[21] M. Zambra et al., IEEE Trans. Plasma Sci. 32, 221 (2004).

[22] Handbook of Vacuum Arc Science and Technology, edited by R.L. Boxman, P. Martin, and D. Sanders (Noyes, New Jersey, 1995).

[23] H. R. Wilson and S.C. Cowley, Phys. Rev. Lett. 92, 175006 (2004).

[24] S. Mar et al., J. Phys. B 33, 1169 (2000).

[25] H. R. Griem, Plasma Spectroscopy (McGraw-Hill, New York, 1964).

[26] A. Ferrari, Annu. Rev. Astron. Astrophys. 36, 539 (1998).

[27] Y. Uchida et al., Astrophys. J. 600, 88 (2004).

[28] M. S. Wheatland, Astrophys. J. 532, 616 (2000). 